\title{
ON OUTER MEASURES AND SEMI-SEPARATION OF LATTICES
}

\section{ROBERT W. SCHUTZ}

P.O. Box 1149

West Babylon, N.Y. 11704 U.S.A.

(Received October 13, 1992 and in revised form September 21, 1993)

\begin{abstract}
This present paper is concerned with set functions related to $\{0,1\}$ two valued measures . These set functions are either outer measures or have many of the same characteristics. We investigate their properties and look at relations among them. We note in particular their association with the semiseparation of lattices.
\end{abstract}

To be more specific, we define three set functions $\mu^{\prime \prime}, \mu^{\prime}$, and $\bar{\mu}$ related to $\mu \varepsilon I(\mathbf{L})$ the $\{0,1\}$ two valued set functions defined on the algebra generated by the lattice of sets $\mathbf{L}$ st $\mu$ is a finitely additive monotone set function for which $\mu(\varnothing)=0$. We note relations among them and properties they possess. In particular necessary and sufficient conditions are given for the semi-separation of lattices in terms of equality of set functions over a lattice of subsets.

Finally the notion of I-lattice is defined, we look at some properties of these with certain other side conditions assume, and end with an application involving semi-separation and I-lattices.

KEY WORDS AND PHRASES.Two valued measures, regular measures, sigma-smooth measures, premeasure, lattice, delta lattice, lindelof, separation, semi-separation, regular, normal, complement generated, I-lattice.

1980 AMS SUBJECT CLASSIFICATION CODES.28A60, $28 A 32$.

\section{1) INTRODUCTION}

In this paper we consider set functions that are related to a measure $\mu$, namely, $\mu^{\prime}, \mu^{\prime \prime}, \mu^{*}$ and also some associated premeasures. We will investigate some of their properties and look at relations among them, and note in particular their association with semi-separation.

To be more precise let $X$ be an abstract set and $L$ a lattice of sets containing $X$ and $\varnothing$. Then for $\mu \varepsilon I(L)$, the two valued $\{0,1\}$ finitely additive non-trivial measures defined on $A(L)$ the algebra generated by the lattice $\mathbf{L}$, we define $\mu^{\prime}$, and note that it is a finitely subadditive "outer measure". (See section 2 for notations and terminology, sections 3 for definitions of $\mu^{\prime}, \mu^{\prime \prime}$.) We also prove that a) If $L$ is regular $S(\mu)=S\left(\mu^{\prime}\right)$.b) $S \mu^{\prime}=\left\{E \mid X \supseteq E\right.$ and either $E \supseteq L$ or $E^{\prime} \supseteq L$ where $\mu(L)=1$ for $\left.L \varepsilon L\right\}$ where $S \mu^{\prime}$ are $\mu^{\prime}-$ measurable sets.c) $L \mu=\left\{L \varepsilon L \mid \mu(L)=\mu^{\prime}(L)\right\}$ is a lattice. d) If $\mu_{1}, \mu_{2} \varepsilon I(L)$ and $\mu_{1} \leq \mu_{2}$ (L) then $\mathrm{L} \mu_{2} \supseteq \mathrm{L} \mu_{1}$.e) $\mathrm{S} \mu^{\prime} \cap \mathrm{L}=\mathrm{L} \mu$.

We also define $\mu^{\prime \prime}$ for $\mu \varepsilon I\left(\sigma^{*}, \mathbf{L}\right)$ and prove that it is a countably sub-additive outer measure. We then prove that the collection of measurable sets $S \mu "=\left\{E \mid X \supseteq E\right.$ st $E \supseteq L_{n} n=1,2, \ldots, \mu\left(L_{n}\right)=1$ all $n L_{n} \varepsilon L$ or $E^{\prime} \supseteq L_{n} n=1,2, \ldots, \mu\left(L_{n}\right)=1$ all $\left.n L_{n} \varepsilon L\right\}$.

Then we prove the following relations hold among $\mu^{\prime}$ and $\mu^{\prime \prime}$; a) $\mu \leq \mu^{\prime \prime} \leq \mu^{\prime}$ (L) $\mu^{\prime \prime} \leq \mu=\mu^{\prime}$ (L') .b) If $\mu \varepsilon I\left(\sigma^{*}, \mathbf{L}\right)$ and $\mathbf{L} \operatorname{cg}$ then $\mu^{\prime \prime}=\mu^{\prime}$ on $\left.\mathbf{L}^{\prime} . c\right)$ If $\mu \varepsilon I\left(\sigma^{*}, \mathbf{L}\right)$ and $\mu=\mu^{\prime \prime}=\mu^{\prime}$ on $\mathbf{L}$ then $\mu \varepsilon \operatorname{IR}(\sigma, \mathbf{L})$.d) $\mu \varepsilon I \$(\mathbf{L})$ for $\mu \varepsilon I\left(\sigma^{*}, \mathbf{L}\right)$ iff $\left.\mu^{\prime}=\mu^{\prime \prime}\left(\mathbf{L}^{\prime}\right) . e\right)$ Finally after defining $\tilde{\mu}$ another finitely subadditive measure with $\mu \varepsilon I(\mathbf{L})$ (see section 4 ) we have the following. If $L_{2} \supseteq L_{1}$ then $L_{1}$ semi-separates $L_{2}$ iff $\mu^{\prime}=\tilde{\mu}$ on $\mathbf{L}_{2}$ for $\mu \varepsilon \operatorname{IR}\left(\mathbf{L}_{2}\right)$. 
In the fourth and final section we define I-lattices. If $\pi \varepsilon \Pi(\sigma, L)$ (see sections four and two for definitions) then there exists a $\mu \varepsilon \operatorname{IR}(\sigma, \mathbf{L})$ st $\pi \leq \mu$ (L) holds, and we prove in particular the following.a) If $L$ is an I-lattice as well as a delta lattice and $\operatorname{I}(\sigma *, L)=\operatorname{IR}(\sigma, \mathbf{L})$ then $L$ is complemented.b) If $L_{1}, L_{2}$ are lattices such that $\mathbf{L}_{2} \supseteq \mathbf{L}_{1}, \mathbf{L}_{1}$ a delta lattice, and for every $\mu \varepsilon I R\left(\sigma, \mathbf{L}_{1}\right) \mu^{*}=\tilde{\mu}\left(\mathbf{L}_{2}\right)$ then $\mathbf{L}_{1}$ semiseparates $\mathbf{L}_{2}$.

\section{2)BASIC NOTATION AND TERMINOLOGY}

In this section we introduce notation and terminology that will be used throughout the paper. We also introduce background material for the readers convenience and references to further background material.Our notation is consistent with Alexandrov [1], Frolik [4], Grassi [5], and Szeto [7].

Let $X$ be an abstract set, and $L$ a lattice of subsets of $X$ st $X, \emptyset \varepsilon L$.A delta lattice is one that is closed under countable intersections and the delta lattice generated by $\mathbf{L}$ is denoted by $\delta(\mathbf{L})$. $\ln$ addition $\mathbf{L}$ is complement generated iff for every element $L \varepsilon L L=\cap L_{n}{ }^{\prime}$ where the $L_{n} \varepsilon L, n=1,2, \ldots$, and the prime will denote complement throughout.A tau lattice is one that is closed under arbitrary intersections, and the tau lattice generated by $\mathbf{L}$ is denoted by $\tau \mathbf{L}$.A(L) will denote the algebra generated by the lattice $\mathbf{L} . \sigma(\mathbf{L})$ denotes the $\sigma$-algebra generated by $\mathbf{L}$.

Let $L_{1}, \mathbf{L}_{2}$ be two lattices such that $\mathbf{L}_{2} \supseteq \mathbf{L}_{1}, \mathbf{L}_{1}$ semi-separates (ss) $\mathbf{L}_{2}$ if for $L_{1} \varepsilon \mathbf{L}_{1}, \mathbf{L}_{2} \varepsilon \mathbf{L}_{2}$, and $\mathrm{L}_{1} \cap \mathrm{L}_{2}=\varnothing$ then there exists $\tilde{\mathrm{L}}_{1} \varepsilon \mathrm{L}_{1}$ st $\mathrm{L}_{1} \sim \mathrm{L}_{2}$ and $\mathrm{L}_{1} \cap \tilde{\mathrm{L}}_{1}=\varnothing$.

Let $I(L)$ denote the set of non-trivial two valued $\{0,1\}$ finitely additive measures on the algebra generated by $\mathbf{L}$, and let $I\left(\sigma^{*}, \mathbf{L}\right)$ denote those elements of $I(L)$ that are sigma-smooth on $L$, i.e. $\left\{L_{n} \mid \varepsilon \mathbf{L}\right.$ , $L_{n} \downarrow \varnothing$ and $\mu \varepsilon I(\sigma *, \mathbf{L})$ then $\lim \mu\left(L_{n}\right)=()$. I $(\sigma, \mathbf{L})$ denotes those elements if $I(\mathbf{L})$ that are sigma-smooth on $A(L)$, i.e if $\left\{A_{n}\right\} \varepsilon A(L), A_{n} \downarrow \varnothing$ and $\mu \varepsilon I(\sigma, L)$ then $\lim \mu\left(A_{n}\right)=0$. This is equivalent to countable additivity of $\mu$ on $A(L)$.IR(L) will stand for the measures on $A(L)$ that are $\mathbf{L}$-regular, i.e. $\mu \varepsilon \operatorname{IR}(\mathbf{L})$, $\mu(A)=\sup \mu(L), L \varepsilon L, A \supseteq L$ and $A \varepsilon A(L)$.This is equivalent to $\mu$ being $L$-regular just on $L$ '. IR( $\sigma, L)$ will denote those measures that sigma-smooth and $\mathbf{L}$-regular on $A(L)$. The obvious relations hold $I(L) \supseteq I(\sigma *$, $\mathbf{L}) \supseteq \mathrm{I}(\sigma, \mathbf{L}) \supseteq \operatorname{IR}(\sigma, \mathbf{L})$ and $\mathrm{I}(\mathbf{L}) \supseteq \operatorname{IR}(\mathbf{L})$. The support of a measure $S(\mu), \mu \varepsilon I(\mathbf{L})$ is defined as $S(\mu)=\cap\{L \varepsilon L \mid \mu(L)=1\}$.

A lattice is said to be disjunctive if for $x \varepsilon X, L \varepsilon L, x \notin L$ then there exists $\tilde{L} \varepsilon \mathbf{L}$ st $x \varepsilon \tilde{\mathrm{L}}$ and $\mathbf{L} \cap \tilde{\mathrm{L}}=\emptyset . \mathbf{L}$ is said to be regular if for $x \varepsilon X, L \varepsilon L, x \notin L$ then there exists $L_{1}, L_{2} \varepsilon L$ st $x \varepsilon L_{1}^{\prime}, L_{2}^{\prime} \supseteq L_{\text {and }}$ $L_{1}{ }^{\prime} \cap L_{2}^{\prime}=\varnothing . L$ is normal if for $L_{1}, L_{2} \varepsilon L$ and $L_{1} \cap L_{2}=\varnothing$, there exists $L_{3}, L_{4} \varepsilon L$ st $L_{3}^{\prime} 2_{1} L_{1} L_{4}^{\prime} 2_{3}^{\prime}$ and $\mathrm{L}_{3} \cap \mathrm{L}_{4}^{\prime}=\emptyset . \mathrm{L}$ is $\underline{\text { lindelof }}$ if for $\left\{\mathrm{L}_{\alpha}\right\} \varepsilon \mathrm{L} \alpha \varepsilon \Lambda$ an arbitriary index set and $\cap \mathrm{L}_{\alpha}=\emptyset \alpha \varepsilon \Lambda$ then there exists a contable subindexing such that $\cap L_{\alpha_{i}}=\varnothing i=1,2, \ldots, . L$ is countably compact if for any $\left\{L_{n}\right\} \varepsilon L$ and $\cap L_{n}=\varnothing n=1,2, \ldots$, there exists a finite subindexing such that $\cap L_{n i}=\varnothing i=1,2, \ldots, N$.

Note. For $\mu_{1}, \mu_{2} \varepsilon I(L)$ we write $\mu_{1} \leq \mu_{2}(\mathbf{L})$ if $\mu_{1}(L) \leq \mu_{2}(L)$ for all $L \varepsilon L$.

By a premeasure is meant a set function $\pi$ defined on $L$ st a) $\pi . L \rightarrow\{0,1\}$, $\pi$ non trivial and $\pi(\emptyset)=0 . b) \pi(A \cap B)=\pi(A) \pi(B) A, B \varepsilon L . c) \pi$ is monotonic. The set of all such premeasures is denoted by $\Pi(L)$. By $\Pi(\sigma, L)$ we mean those $\pi \varepsilon \prod(L)$ st $\pi\left(A_{n}\right)=1$ all $n$ implies that $\cap A_{n} \neq \emptyset n=1,2, \ldots$, and $A_{n} \varepsilon L$.

We note some measure equivalence of topologicial properties.

1) $L$ is disjunctive iff for all $x \varepsilon X, \mu_{X} \varepsilon \operatorname{IR}(\sigma, L)$ where $\mu_{X}$ is the point measure, i.e. $\mu_{X}(A)=1$ if $x \varepsilon A$, $\mu_{\mathrm{X}}(\mathrm{A})=0 \mathrm{x} \notin \mathrm{A} \mathrm{A \varepsilon A}(\mathrm{L})$.

2) $\mathbf{L}$ is regular iff $\mu \leq \mu_{1}(\mathbf{L}) \mu, \mu_{1} \varepsilon \mathrm{I}(\mathbf{L})$ implies $S(\mu)=S\left(\mu_{1}\right)$.

3) $\mathbf{L}$ is normal iff $\mu \varepsilon I(\mathbf{L}), \mu_{1}, \mu_{2} \varepsilon \operatorname{IR}(\mathbf{L}), \mu \leq \mu_{1}(\mathbf{L})$, and $\mu \leq \mu_{2}(\mathbf{L})$ implies that $\mu_{1}=\mu_{2}$.

4) $\mathbf{L}$ is countably compact iff $\mu \varepsilon I(L)$ implies that $\mu \varepsilon I\left(\sigma^{*}, \mathbf{L}\right)$.

5) $L$ is lindelof iff $\pi \varepsilon \prod(\sigma, L)$ implies $S(\pi) \neq \emptyset$. Where $S(\pi)=\cap\{L \varepsilon L I \pi(L)=1\}$.

The following facts will be used in this paper.There exists a one to one correspondence between prime $\mathbf{L}$-filters and elements of $\mathbf{I}(\mathbf{L})$, and a one to one correspondence between $\mathbf{L}$-ultrafilters and elements of $\operatorname{IR}(\mathbf{L})$. This correspondence is set up by letting $\mu \varepsilon I(L)$ and $H=\{L \varepsilon L \mid \mu(L)=1\}$.Then $H$ is a prime $L$-filter and conversely if $H$ is a prime $L$-filter there exists a measure $\mu \varepsilon I(L)$ associated with $H$ st $\mu(L)=1$, iff $L \varepsilon H$ .Also if $\mu \varepsilon \operatorname{IR}(\mathbf{L})$ then $\mathrm{H}$ is an $\mathbf{L}$-ultrafilter and conversely if $\mathrm{H}$ is an $\mathbf{L}$-ultrafilter then there exists a 
$\mu \varepsilon I R(L)$ such that $\mu(L)=1$ iff $L \varepsilon H$. In addition there exists a one to one correspondence between $\Pi(L)$ and all $L$ filters $F$ given by $\pi \leftrightarrow F$ an $L$ filter iff $\pi(L)=1$ for $L \varepsilon F, F$ an $L$ filter .

DEFINITION 2.1. Let $\mu \varepsilon I(L)$ then for $E$ st $X \supseteq E \mu^{\prime}(E)=\inf \mu\left(L^{\prime}\right)$ and the inf is taken over all LEL and $L^{\prime} \supseteq E$.

DEFINITION 2.2. Let $\mu \varepsilon I(\mathbf{L})$, then $\mu \varepsilon I W(\mathbf{L})$ if $\mu\left(L^{\prime}\right)=1$ LeL implies that there exists a $\tilde{L} \varepsilon \mathbf{L}$ st $L^{\prime} \supseteq \tilde{\mathrm{L}}$ and $\mu^{\prime}(\tilde{\mathrm{L}})=1$.

DEFINITION 2.3. $\mu \varepsilon I \$(L)$ if for $\left\{A_{n}\right\} \varepsilon L A_{n} \downarrow$, and $\cap A_{n}=A \varepsilon L$ then $\lim \mu\left(A_{n}\right)=\mu(A)$. Note. $I(\sigma *, \mathbf{L}) \supseteq I \$(\mathbf{L})$.

DEFINITION 2.4. Let $\mu \varepsilon I(\sigma *, L)$ and for $E$ st $X \supseteq E$ define $\mu "(E)=\inf \Sigma \mu\left(L_{i}{ }^{\prime}\right)$ where the inf is over all $\cup L_{i^{\prime}}, i=1,2, \ldots, L_{i} \varepsilon L$, and $\cup L_{i}{ }^{\prime} \supseteq E$.

Next we consider various sets of measures defined on the algebra generated by a lattice $L$. For example $I(L), I\left(\sigma^{*}, \mathbf{L}\right), I R(L)$ or $I R(\sigma, L)$.Denote such sets by I.Also consider the collection of sets $H(L)$ where $H(L)=\{H(L) \mid L \varepsilon L\}, H(L)=\{\mu \varepsilon|| \mu(L)=1\}$. Then the following hold the . a) $H(A \cap B)=H(A) \cap H(B)$ for A. $B \varepsilon L$.b) $H(A \cup B)=H(A) \cup H(B) A, B \varepsilon L$

c) $H\left(A^{\prime}\right)=H(A)^{\prime}$ for $A \varepsilon L$.d) If $A \supseteq B$ then $H(A) \supseteq H(B) A, B \varepsilon L$.e) If $L$ is disjunctive (if necessary) then $H(A) \supseteq H(B)$ implies $A \supseteq B, A, B \varepsilon L . f)$ The collection $H(L)$ is a lattice and $H(A(L))=A(H(L))$.

We will assume that in discussing $H(L)$ for convenience, that $L$ is disjunctive, although it will be clear that this assumption is not always needed.

If $\mu \varepsilon I$ then define a measure on $A(H(L)) \hat{\mu} \varepsilon I(H(L))$ by $\hat{\mu}(H(A))=\mu(A)$ for $A \varepsilon A(L)$. Conversely if $\hat{\mu} \varepsilon I(H(L))$ define a measure on $\mu \varepsilon I$ by $\mu(A)=\hat{\mu}(H(A)) H(A) \varepsilon A(H(L))$. Then the following hold.

THEOREM 2.1. If $L$ is disjunctive (if necessary) then there exists a one to one correspondence between the sets $I$ and $I(A(L))$ given by $\mu \leftrightarrow \hat{\mu}$. Further $\mu \varepsilon I$ is $\sigma$-smooth or $L$-regular iff $\hat{\mu} \varepsilon I(H(L))$ is $\sigma$-smooth or $\mathrm{H}(\mathrm{L})$ regular respectively.

If $\mathrm{I}=\mathrm{IR}(\mathbf{L})$ we let $\mathrm{H}(\mathbf{L})=\mathrm{W}(\mathbf{L})$.

If $I=I R(\sigma, L)$ we let $H(L)=W(\sigma, L)$.

We define $\mu^{*}$ for $\mu \varepsilon I(\sigma, L)$ such that if $X \supseteq E \mu^{*}(E)=i n f \Sigma \mu\left(A_{i}\right), A_{i} \varepsilon A(L), \cup A_{i} \supseteq E i=1,2, \ldots$, As is well known $\mu^{*}$ is an outer measure, the $\mu^{*}$ measurable sets form a $\sigma$-algebra containing $\sigma(\mathbf{L})$ and the restriction of $\mu^{*}$ to $A(\mathbf{L})$ agrees with $\mu$.

Further related material can be found in Camacho [2], Eid [3], and Huerta |6] .

3) DEFINITIONS OF $\mu^{\prime}, \mu^{\prime \prime}$ AND THEIR BASIC PROPERTIES

In this section we examine two set functions $\mu^{\prime \prime}, \mu^{\prime}$ that are related to a measure $\mu \varepsilon I(L)$ or $\mu \varepsilon I\left(\sigma^{*}, \mathbf{L}\right)$ .First we look at at $\mu^{\prime \prime}$ which is genuine countably subadditive outer measure and is defined for all $\mu \varepsilon I(\sigma *, \mathbf{L})$.We also define $\mu^{\prime}$ which is finitely subadditive "outer measure" defined for $\mu \varepsilon I(L)$. We then investigate some of the properties of these set functions and relationships that hold for them. We finally consider conditions for one lattice to semi-separate another in terms of $\mu^{\prime}$ and $\tilde{\mu}$ another related set function.

We first have the following theorem involving $\mu^{\prime \prime}$ and $\mu^{\prime}$.

THEOREM 3.1. a) Let $\mu \varepsilon I\left(\sigma^{*}, \mathbf{L}\right), \quad \mu^{\prime \prime}$ is an outer measure on $X$. b)Let $\mathbf{S} \mu^{\prime \prime}$ denote the $\mu^{\prime \prime}$ measurable sets where $\mu \varepsilon I\left(\sigma^{*}, L\right)$, then $S \mu^{\prime \prime}=\left\{E, X \supseteq E \mid E \supseteq \cap L_{n}\right.$ st $\mu\left(L_{n}\right)=1$ all $n$, or $E^{\prime} \supseteq \cap L_{n}$ where for all $\left.n \mu\left(L_{n}\right)=1 L_{n} \varepsilon L\right\}$. c) For $\mu \varepsilon I(L), \mu^{\prime}$ is a finitely subadditive "outer measure". d) Let $S \mu^{\prime}$ denote the $\mu^{\prime}$ measurable sets where $\mu \varepsilon I(L)$, then $S \mu^{\prime}=\left\{E, X \supseteq E\right.$ । and either $E \supseteq \tilde{L}$ or $E^{\prime} \supseteq \tilde{L}$ where $\mu(\tilde{L})=1 L$, $\tilde{L} \varepsilon L\}$. e) If $L$ is a regular lattice, then $S(\mu)=S\left(\mu^{\prime}\right)$, where $S\left(\mu^{\prime}\right)$ is the support of the set function $\mu^{\prime}$, $S\left(\mu^{\prime}\right)=\bigcap\left\{L \varepsilon L I \mu^{\prime}(L)=1\right\}$.

We will only prove parts $b$ and e since the other parts follow readily or are similiar in spirit.

Proof . b)Let $E \varepsilon S \mu^{\prime \prime}$ then $\mu^{\prime \prime}(A)=\mu^{\prime \prime}(A \cap E)+\mu^{\prime \prime}\left(A \cap E^{\prime}\right)$ for all $A$ st $X \supseteq A$.In particuliar let $A=X$ then $1=\mu^{\prime \prime}(E)+\mu^{\prime \prime}\left(E^{\prime}\right)$ and either $\mu^{\prime \prime}(E)=1$ and $\mu^{\prime \prime}\left(E^{\prime}\right)=0$ or $\mu^{\prime \prime}\left(E^{\prime}\right)=1$ and $\mu^{\prime \prime}(E)=0$.Assume $\mu^{\prime \prime}(E)=0$ then $\mu^{\prime \prime}(E)=\inf \Sigma \mu\left(L_{n^{\prime}}\right), \cup L_{n^{\prime}} \supseteq E, n=1,2, \ldots$, and $L_{n} \varepsilon L$. Thus $\mu\left(L_{n}{ }^{\prime}\right)=0$ or $\mu\left(L_{n}\right)=1$ all $n$ and $E^{\prime} \supseteq \cap L_{n}$. Similiarly if $\mu^{\prime \prime}\left(E^{\prime}\right)=0$ then $E \supseteq \cap L_{n}$ and $\mu\left(L_{n}\right)=1$ all $n, n=1,2, \ldots, \infty$. 
Proof. e) Since $\mu \leq \mu^{\prime}$ on $L$ then $S(\mu) \supseteq S\left(\mu^{\prime}\right)$. Suppose that $S(\mu) \neq S\left(\mu^{\prime}\right)$.Let $x \varepsilon S(\mu)$ and $x \notin S\left(\mu^{\prime}\right)$. Then there exists a $L \varepsilon L$ st $\mu^{\prime}(L)=1, x \notin L$, and $\mu(L)=()$. Since $L$ is regular there exists $L_{1}, L_{2} \varepsilon L$ st $x \varepsilon L_{1}{ }^{\prime}$, $\mathrm{L}_{2}{ }^{\prime} \supseteq \mathrm{L}$ and $\mathrm{L}_{1}{ }^{\prime} \cap \mathrm{L}_{2}{ }^{\prime}=\emptyset \cdot \mu\left(\mathrm{L}_{2}{ }^{\prime}\right)=\mu^{\prime}\left(\mathrm{L}_{2}{ }^{\prime}\right)=1, \mathrm{~L}_{1} \cup \mathrm{L}_{2}=\mathrm{X}, \mu\left(\mathrm{L}_{2}\right)=0$ therefore $\mu\left(\mathrm{L}_{1}\right)=\mu^{\prime}\left(\mathrm{L}_{1}\right)=1$ and $x \notin L_{1}$. Thus $x \notin S(\mu)$, a contradiction. $S(\mu)=S\left(\mu^{\prime}\right)$.

DEFINITION 3.1. Let $L \mu=\left\{\mathrm{L} \varepsilon \mathrm{L} \mid \mu(\mathrm{L})=\mu^{\prime}(\mathrm{L})=1\right\}$

THEOREM 3.2. If $\mu_{1}, \mu_{2} \varepsilon I(\mathbf{L})$ and if $\mu_{1} \leq \mu_{2}$ (L) then $\mathbf{L} \mu_{1} \supseteq \mathbf{L} \mu_{2}$.

PROOF.Let $L_{1} \varepsilon L \mu_{1}$ then $\mu_{1}\left(L_{1}\right)=\mu_{1}{ }^{\prime}\left(L_{1}\right)$. If $\mu_{1}{ }^{\prime}\left(L_{1}\right)=\mu_{1}\left(L_{1}\right)=1$ then $\mu_{2}\left(L_{1}\right)=1$ and since $\mu_{2} \leq \mu_{2}{ }^{\prime}$ on $L \mu_{2}{ }^{\prime}\left(L_{1}\right)=1$ and $\mu_{2}\left(L_{1}\right)=\mu_{2}\left(L_{1}\right)$ and $L_{1} \varepsilon L_{2}$. Now suppose $\mu_{1}\left(L_{1}\right)=\mu_{1}{ }^{\prime}\left(L_{1}\right)=0$ $\mu_{2}\left(L_{1}\right)=0$ and $\mu_{2}{ }^{\prime}\left(L_{1}\right)=1$. Then $\mu_{2}{ }^{\prime}\left(L_{1}\right)=\inf \mu_{2}\left(\tilde{L}^{\prime}\right)=1$ where $\tilde{L}^{\prime} \supseteq L_{1}$. But since $\mu_{1} \leq \mu_{2}(L)$ then $\mu_{2} \leq \mu_{1}$ on $L^{\prime}$ and $0=\inf \mu_{1}\left(\tilde{L}^{\prime}\right)=\mu_{1}{ }^{\prime}\left(L_{1}\right) \geq \inf \mu_{2}\left(\tilde{L}^{\prime}\right)=\mu_{2}{ }^{\prime}\left(L_{1}\right)=1$ a contradiction. Thus $\mu_{2}{ }^{\prime}\left(L_{1}\right)=0$ and $\mu_{2}\left(L_{1}\right)=\mu_{2}{ }^{\prime}\left(L_{1}\right)=0$.If $\mu_{1}{ }^{\prime}\left(L_{1}\right)=\mu_{1}\left(L_{1}\right)=0$ and $\mu_{2}\left(L_{1}\right)=1$ then $\mu_{2}{ }^{\prime}\left(L_{1}\right)=1$, but $\mu_{1}{ }^{\prime} \geq \mu_{2}{ }^{\prime}\left(L^{\prime}\right)$, a contradiction. Thus $\mu_{2}\left(L_{1}\right)=\mu_{2}^{\prime}\left(L_{1}\right)=()$. This implies then that $L \mu_{2} \supseteq L_{1} \mu_{1}$.

THEOREM 3.3. Let $\mu \varepsilon I(L)$, then $S \mu^{\prime} \cap L=L \mu$.

Proof. Let $L \varepsilon S \mu^{\prime} \cap L$ then $\mu^{\prime}(E)=\mu^{\prime}(L \cap E)+\mu^{\prime}\left(E \cap L^{\prime}\right)$ for all $E$ st $X \supseteq E$.In particuliar for $E=X$ $1=\mu^{\prime}(L)+\mu^{\prime}\left(L^{\prime}\right)$. If $\mu^{\prime}\left(L^{\prime}\right)=1$ then $\mu^{\prime}(L)=0$, and since $\mu^{\prime} \geq \mu(L) \mu(L)=0$ and $\mu(L)=\mu^{\prime}(L)=0$. If $\mu^{\prime}(L)=1$ then $\mu^{\prime}\left(L^{\prime}\right)=0$ which implies that $\mu\left(L^{\prime}\right)=0$ since $\mu=\mu^{\prime}$ on $\left(L^{\prime}\right)$ or $\mu(L)=1$, and $\mu(L)=\mu^{\prime}(L)=1$. Thus in both cases $L \varepsilon L \mu$ and $L \mu \supseteq S \mu \prime \cap L$.

Conversely let $L \varepsilon L \mu, L \mu$ is contained in $\mathbf{L}$. Need to prove that $S \mu \nmid \mathbf{L} \mu$. For $\tilde{L} \varepsilon \mathbf{L} \mu$ and $\mathbf{L} \supseteq \tilde{\mathbf{L}}$, assume that $\mu(\tilde{\mathrm{L}})=0$ for all such $\tilde{\mathrm{L}}$. In particular it holds for $\tilde{\mathrm{L}}=\mathrm{L}$ or $\mu(\mathrm{L})=0$. But since $\mathrm{L} \varepsilon \mathrm{L} \mu$ $\mu^{\prime}(L)=0 . \mu^{\prime}(L)=\inf \mu\left(L_{1}^{\prime}\right)=0$ for $L_{1}^{\prime} \supseteq L$ or there exists a $L_{1} \varepsilon L$ st $\mu\left(L_{1}^{\prime}\right)=()$ or $\mu\left(L_{1}\right)=1, L^{\prime} \supseteq L_{1}$ thus $L \varepsilon S \mu^{\prime}$.If $\mu(\mathrm{L})=1 \mathrm{~L} \supseteq \mathrm{L}$ and $\mathrm{L} \varepsilon S \mu^{\prime}$. Thus $S \mu^{\prime} \cap \mathrm{L}=\mathrm{L} \mu$.

COROLLARY 3.1. $L \mu$ is a lattice.

PROOF. Since by theorem 3.3 $S \mu^{\prime} \cap L=L \mu, S \mu^{\prime}, L$ are lattices and the intersection of two lattices is a lattice the result follows.

THEOREM 3.4. Let $\mu \varepsilon I(\sigma *, \mathbf{L})$. Then $\mu \leq \mu^{\prime \prime} \leq \mu^{\prime}(\mathbf{L})$ and $\mu^{\prime \prime} \leq \mu=\mu^{\prime}\left(\mathbf{L}^{\prime}\right)$.

PROOF. It is clear that $\mu^{\prime \prime} \leq \mu=\mu^{\prime}\left(L^{\prime}\right)$ and that $\mu^{\prime \prime} \leq \mu^{\prime}$ everywhere. Thus we must just show that $\mu \leq \mu$ " (L).Assume not then there exists $L \varepsilon L$ st $\mu(L)=1$ and $\mu^{\prime \prime}(L)=0$. Thus $\mu^{\prime \prime}(L)=\inf \Sigma \mu\left(L_{i}{ }^{\prime}\right)=0$ and thus there exists $\cup L_{i^{\prime}} \supseteq L i=1,2, \ldots$, st $L_{i} \varepsilon L$ and $\mu\left(L_{i}{ }^{\prime}\right)=0$ all $i$ or $\mu\left(L_{i}\right)=1$ all $i$.Then $L^{\prime} \supseteq \cap L_{i}$ and

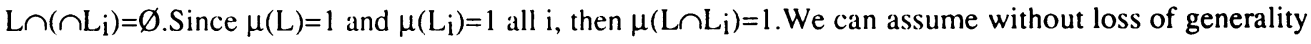
that $\left\{L \cap L_{i}\right\} \downarrow \emptyset$, then since $\mu \varepsilon I(\sigma *, L), \quad 0=\lim \mu\left(L \cap L_{i}\right)=1$, a contradiction. Thus $\mu(L)=0$ and $\mu \leq \mu^{\prime \prime} \leq \mu^{\prime}$ (L).

THEOREM 3.5. If $\mu \varepsilon I(\sigma *, L)$ and if $\mu=\mu^{\prime}=\mu^{\prime \prime}$ on $\mathbf{L}$ then $\mu \varepsilon I R(\sigma, \mathbf{L})$.

PROOF. Let $\mu\left(L^{\prime}\right)=1 \quad L \varepsilon L$ then $\mu(L)=0$ and $\mu^{\prime}(L)=0$. Thus there exists a $\tilde{L} \varepsilon L$ st $\tilde{L}^{\prime} \supseteq \mathbf{L}$ and $\mu\left(\tilde{L}^{\prime}\right)=0$ or $\mu(\tilde{L})=1$ and $L^{\prime} \supseteq \tilde{L}$. Therefore $\mu \varepsilon \operatorname{IR}(\sigma, L)$.

THEOREM 3.6. Let $\mu \varepsilon I(\sigma *, \mathbf{L})$ then $\mu^{\prime}=\mu^{\prime \prime}\left(\mathbf{L}^{\prime}\right)$ iff $\mu \varepsilon I \$(\mathbf{L})$.

PROOF. Let $\mu \varepsilon I\left(\sigma^{*}, \mathbf{L}\right)$ and $\mu^{\prime}=\mu^{\prime \prime}\left(\mathbf{L}^{\prime}\right)$.Assume that $\mu \notin I \$(\mathbf{L})$ and let $\cap A_{n} \downarrow A, A, A_{n} \varepsilon L$ such that $\mu\left(A_{n}\right)=1$ all $n$ and $\mu(A)=0$. Then $\mu\left(A^{\prime}\right)=1 \mu\left(A^{\prime}\right)=\mu^{\prime}\left(A^{\prime}\right)=\mu^{\prime \prime}\left(A^{\prime}\right)=1$ by hypothesis. But $\mu^{\prime \prime}\left(A^{\prime}\right)=\mu^{\prime \prime}\left(\cup A_{n^{\prime}}\right)=\Sigma \mu\left(A_{n}{ }^{\prime}\right)=0$ since $\mu\left(A_{n}{ }^{\prime}\right)=0$ all $n$, a contradiction. $\mu \varepsilon I \$(L)$.

Conversely let $\mu \varepsilon I \$(L)$ and assume that $\mu^{\prime \prime} \leq \mu=\mu^{\prime}$ on $L^{\prime}$. Let $\mu "\left(L^{\prime}\right)=0$ then there exists $\cup L_{i}{ }^{\prime} 2 L^{\prime} L$, $L_{i} \varepsilon L i=1,2, \ldots$, st $\mu\left(L_{i}{ }^{\prime}\right)=0$ all $i$ or $\mu\left(L_{i}\right)=1$ all $i$, and $L \supseteq \cap L_{i}$, also $L=\cap\left(L \cup L_{i}\right)$

.We can assume without loss of generality that $\left(L \cup L_{i}\right\} \downarrow L$ then $\mu(L)=\inf \mu\left(L \cup L_{i}\right)=i n f 1=1$ since $\mu \varepsilon I \$(L)$.Then $\mu\left(L^{\prime}\right)=\mu^{\prime}\left(L^{\prime}\right)=0$ thus $\mu^{\prime}=\mu^{\prime \prime}$ on $L^{\prime}$.

We now look at another class of measures we defined previously, IW(L).

THEOREM 3.7. If $\mu \varepsilon I \$(\mathbf{L})$ and if the lattice $\mathbf{L}$ is cg the $\mu \varepsilon I W(L)$.

PROOF. Suppose that $L \varepsilon L$ and $\mu^{\prime}\left(L^{\prime}\right)=\mu\left(L^{\prime}\right)=1$.Then from theorem $3.6 \mu \varepsilon I \$(L)$ implies $\mu^{\prime}=\mu^{\prime \prime}$ on $L^{\prime}$, hence $\mu^{\prime \prime}\left(L^{\prime}\right)=1$. Since $L_{\text {is }}$ cg then $L^{\prime}=\cup L_{i} L_{i} \varepsilon L i=1,2, \ldots$, and $1=\mu^{\prime \prime}\left(\cup L_{i}\right) \leq \Sigma \mu "\left(L_{i}\right)$, since $\mu^{\prime \prime}$ is an outer measure. Thus $\mu^{\prime \prime}\left(L_{i}\right)=1$ for some $i$.Then because $\mu^{\prime} \geq \mu^{\prime \prime}(L) \mu^{\prime}\left(L_{i}\right)=\mu^{\prime \prime}\left(L_{i}\right) L^{\prime} \supseteq L_{i}$. Thus $\mu \varepsilon \operatorname{IW}(\mathbf{L})$. 
THEOREM 3.8. IW(L) $\supseteq \operatorname{IR}(\mathbf{L})$ and if $\mathbf{L}$ is nomal $\operatorname{IR}(\mathbf{L})=\operatorname{IW}(\mathbf{L})$.

PROOF. Let $\mu \varepsilon I R(L)$ and let $\mu\left(L^{\prime}\right)=1 L^{\prime} \varepsilon L^{\prime}$. Then since $\mu \varepsilon I R(L)$ there exists a $\tilde{L} \varepsilon L^{\prime}$ st $L^{\prime} \supseteq L^{\sim}$ st $\mu(\tilde{L})=1$ and since $\mu^{\prime} \geq \mu(\mathbf{L}) \mu^{\prime}(\tilde{L})=1$ and thus $\mu \varepsilon \operatorname{IW}(\mathbf{L})$.

Assume now that $\mathbf{L}$ is normal and let $\mu \varepsilon \mathrm{IW}(\mathbf{L})$ and $\mu\left(L^{\prime}\right)=1 \quad L \varepsilon L$. Then since $\mu \varepsilon I W(L) \mu^{\prime}(\tilde{L})=1$ and $L^{\prime} \supseteq \tilde{L}, \quad \tilde{L} \varepsilon \mathbf{L}$ and $L \cap \tilde{L}=\emptyset$. Since $L$ is normal there exists $L_{1}, L_{2} \varepsilon \mathbf{L}$ st $L_{1}{ }^{\prime} \supseteq L_{2} L_{2} \supseteq \tilde{L}$ and $\mathrm{L}_{1}{ }^{\prime} \cap \mathrm{L}_{2}{ }^{\prime}=\emptyset \cdot \mu\left(\mathrm{L}_{2}{ }^{\prime}\right)=1$ since $\mathrm{L}_{2}{ }^{\prime} \supseteq \tilde{\mathrm{L}}$ and $\mu^{\prime}(\tilde{\mathrm{L}})=1$ implies that $\mu^{\prime}\left(\mathrm{L}_{2}{ }^{\prime}\right)=\mu\left(\mathrm{L}_{2}{ }^{\prime}\right)=1$, thus $\mu\left(\mathrm{L}_{2}\right)=0$, and since $L_{1} \cup L_{2}=X$, then $\mu\left(L_{1}\right)=1$ and $L^{\prime} \supseteq L_{1}$ implies that $\mu \varepsilon I R(L)$.

REMARK. By theorems 3.7 and 3.8 if $\mathbf{L}$ is cg and normal then $\mu \varepsilon I \$(L)$ implies $\mu \varepsilon I R(\sigma, L)$.

THEOREM 3.9. If $\mathbf{L}$ is $\mathrm{cg}$ and $\mu \varepsilon I\left(\sigma^{*}, \mathbf{L}^{\prime}\right)$ implies $\mu \varepsilon I R(\mathbf{L})$.

PROOF. Result is well known see references Camacho |2|, Eid |3], Grassi [5], and Szeto [7].

THEOREM 3.10. If $\mu \varepsilon I\left(\sigma *, \mathbf{L}^{\prime}\right)$ and $\mathbf{L}$ is cg then $\mu=\mu^{\prime}=\mu^{\prime \prime}$ on $\mathbf{L}^{\prime}$.

PROOF. Since $\mu \varepsilon I\left(\sigma *, L^{\prime}\right)$ then $\mu \varepsilon I(L)$ and $\mu=\mu^{\prime}$ on $L^{\prime}$.Assume that $\mu\left(L^{\prime}\right)=\mu^{\prime}\left(L^{\prime}\right)=1$ and since $L$ is cg $L^{\prime}=\cup L_{i} L, L_{i} \varepsilon L i=1,2, \ldots$. Then $\mu^{\prime \prime}\left(L^{\prime}\right) \leq \Sigma \mu^{\prime \prime}\left(L_{i}\right)$ and assume that $\mu^{\prime \prime}\left(L_{i}\right)=()$ all $i$, then $\mu\left(L_{i}\right)=0$ all $i$, and $\mu\left(L_{i}{ }^{\prime}\right)=1$ all $i$. Since $L=\cap L_{i}{ }^{\prime} i=1,2, \ldots$ then $L \cap L^{\prime}=L \cap\left(\cap L_{i}{ }^{\prime}\right)=\varnothing$. We can assume then, without loss of generality that $\left\{L^{\prime} \cap L_{i^{\prime}}\right\} \downarrow \emptyset$. Since $\mu \varepsilon\left(\sigma *, L^{\prime}\right), \lim \mu\left(L^{\prime} \cap L_{i}{ }^{\prime}\right)=0$. But $\mu\left(L^{\prime}\right)=1$ $\mu\left(L_{i}^{\prime}\right)=1$, a contradiction. Thus $\mu^{\prime \prime}\left(L_{i}\right)=1$ for some i. Since $L^{\prime} \supseteq L_{i}$ by the monotone nature of $\mu^{\prime \prime}, \mu^{\prime \prime}\left(L^{\prime}\right)=1$ and $\mu=\mu^{\prime}=\mu^{\prime \prime}$ on $L^{\prime}$.

We now introduce a definition preparatory to presenting our final theorem in this section, relating semiseparation of lattices.

DEFINITION 3.2. Let $\mu \varepsilon I(L)$ and $X \supseteq E$.We define $\tilde{\mu}(E)=\inf \mu(L) L \supseteq E$ and $L \varepsilon L$.

Note that $\mu \sim$ is a finite subadditive "outer measure".

THEOREM 3.11. Let $L_{1}$ and $L_{2}$ be lattices of substs of $X$ st $L_{2} \supseteq L_{1}$.If $L_{1}$ semi-separates $L_{2}$ then $\tilde{\mu}=\mu^{\prime}$ on $L_{2}$ for $\mu \varepsilon \operatorname{IR}\left(L_{1}\right)$. Conversely if for every $\mu \varepsilon \operatorname{IR}\left(L_{1}\right) \tilde{\mu}=\mu^{\prime}$ on $L_{2}$, then $L_{1}$ semi-separates $L_{2}$.

PROOF. Let $L_{1}$ semi-separate $L_{2}$, and look at $\mu^{\prime}\left(L_{2}\right)=\inf \mu\left(L_{1}\right)^{\prime} L_{1}{ }^{\prime} L_{2}, L_{1} \varepsilon L_{1}$ and $L_{2} \varepsilon L_{2}$. Then since $L_{1} \cap L_{2}=\varnothing$ and $L_{1}$ semi-separates $L_{2}$ there exists $\tilde{L}_{1} \varepsilon L_{1}$ st $\tilde{L}_{1} \cap L_{1}=\varnothing$ and $\tilde{L}_{1} \supseteq L_{2}$, or $\mathrm{L}_{1} \supseteq \tilde{\mathrm{L}}_{1}$. Thus $\inf \mu\left(\mathrm{L}_{1}{ }^{\prime}\right) \geq \inf \mu\left(\tilde{\mathrm{L}}_{1}\right) \tilde{\mathrm{L}}_{1} \supseteq \mathrm{L}_{2} \mathrm{~L}_{1}{ }^{\prime} \supseteq \mathrm{L}_{2}$ or $\mu^{\prime} \geq \mu \sim$. Now look at $\tilde{\mu}\left(\mathrm{L}_{2}\right)=\inf \mu\left(\tilde{\mathrm{L}}_{1}\right) \tilde{\mathrm{L}}_{1} \supseteq \mathrm{L}_{2}$ $\tilde{L}_{1 \varepsilon L_{1}}, L_{2} \varepsilon L_{2}$. Assume $\tilde{\mu}\left(L_{2}\right)=0$ then there exists $\tilde{L}_{1} \supseteq L_{2} \tilde{L}_{1} \varepsilon L_{1}$ st $\mu\left(\tilde{L}_{1}\right)=0$ or $\mu\left(\tilde{L}_{1}{ }^{\prime}\right)=1$. Since $\mu \varepsilon \operatorname{IR}\left(L_{1}\right)$ there exists $L_{3} \varepsilon L_{1}$ st $\tilde{L}_{1}{ }^{\prime} \supseteq L_{3} \mu\left(L_{3}\right)=1$ or $\left.\mu\left(L_{3}{ }^{\prime}\right)=0\right) L_{3} \supseteq \tilde{L}_{1} \supseteq L_{2}$ or $\tilde{\mu}\left(L_{2}\right)=\mu^{\prime}\left(L_{2}\right)=0$. Thus $\tilde{\mu}=\mu^{\prime}$ on $\mathbf{L}_{2}$.

Conversely let $\tilde{\mu}=\mu^{\prime}$ on $\mathbf{L}_{2}$ for all $\mu \varepsilon I R\left(\mathbf{L}_{1}\right)$ and assume that $\mathbf{L}_{1}$ does not semi-separate $\mathbf{L}_{2}$. Then there exists $L_{1} \varepsilon L_{1} L_{2} \varepsilon L_{2}$ st $L_{1} \cap L_{2}=\varnothing$ and $\tilde{L}_{1} \cap L_{1} \neq \varnothing$ for all $\tilde{L}_{1} \varepsilon L_{1}$ st $\tilde{L}_{1} \supseteq L_{2}$. Look at $H=\left\{\tilde{L}_{1} \mid\right.$

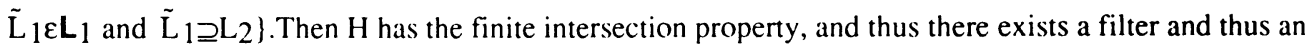
ultrafilter and its associated measure $\mu \varepsilon \operatorname{IR}\left(\mathrm{L}_{1}\right)$ st $\mu\left(\tilde{\mathrm{L}}_{1}\right)=1 \mathrm{~L}_{1} \sim \varepsilon \mathrm{H}$ and since $\mathrm{L}_{1} \cap \tilde{\mathrm{L}}_{1} \neq \emptyset, \mu\left(\mathrm{L}_{1}\right)=1$. Now look at $\mu^{\prime}\left(L_{2}\right)$. Since $L_{1} \cap L_{2}=\varnothing$ then $L_{1}^{\prime} \supseteq L_{2}$ and since $\mu\left(L_{1}\right)=1 \mu\left(L_{1}{ }^{\prime}\right)=0$ and thus $\mu^{\prime}\left(L_{2}\right)=0$.Also $\tilde{\mu}\left(L_{2}\right)=\inf \mu\left(L_{4}\right) L_{4} \supseteq L_{2}$ and $L_{4} \varepsilon L_{1}$. Then since every such $L_{4}$ is a member of $H$ and thus $\tilde{\mu}\left(L_{2}\right)=\inf \mu\left(L_{4}\right)=1$, a contradiction. $L_{1}$ semi-separates $L_{2}$.

\section{4) PROPERTIES OF I-LATTICES AND THEIR RELATIONSHIP TO SEMI-SEPARATION}

In this section we define the notion of an I-lattice and look at necessary and sufficent conditions for an I-lattice to exist such as countable compactness, disjunctiveness and lindelof property to hold.We finally investigate the semi-separation of two lattices $L_{1}, L_{2}$ with $L_{1}$ an I-lattice in terms of outer measures associated with $\mu \varepsilon I\left(\sigma *, L_{1}\right)$.

DEFINITION 4.1. $\mathbf{L}$ is an I-lattice iff for every $\pi \varepsilon \Pi(\sigma, \mathbf{L})$ there exists a $\mu \varepsilon I R(\sigma, \mathbf{L})$ st $\pi \leq \mu(\mathbf{L})$.

DEFINITION 4.2. $L$ is replete iff for every $\mu \varepsilon I R(\sigma, L) S(\mu) \neq \varnothing$.

The results of theorem 4.1 are well known see references Szeto [7]. We prove part $d$ in a more straight forward manner than the above reference shows.

THEOREM 4.1. a)If $\mathbf{L}$ is an I-lattice, and if $\mathbf{L}$ is replete then $\mathbf{L}$ is lindelof. $b$ ) If $\mathbf{L}$ is a countably compact lattice then $L$ is I-lattice. c) If $L$ is a disjunctive lattice and if $L$ is lindelof then $L$ is an I-lattice. d) Suppose $\mathbf{L}$ is disjunctive, then $\operatorname{IR}(\sigma, \mathbf{L}), \tau W(\sigma, \mathbf{L})$ is lindelof iff $\mathbf{L}$ is an I-lattice. 
PROOF. Of part d)Assume that $\mathbf{L}$ is disjunctive. First $W(\sigma, \mathbf{L})$ is lindelof iff $\tau W(\sigma, \mathbf{L})$, thus it is sufficent to prove that $W(\sigma, L)$ is lindelof.Look at $\hat{\pi} \varepsilon \Pi(\sigma, W(\sigma, L))$ then projecting down look at $\pi \varepsilon \Pi(\sigma, L) \pi(L)=\hat{\pi}(W(\sigma, L))$ for $L \varepsilon L$. Since $L$ is disjunctive $I R(\sigma, L) \supseteq\left\{\mu_{x} . x \varepsilon X\right\}$ and if $W\left(\sigma, L_{n}\right) \downarrow \varnothing$, then $L_{n} \downarrow \varnothing$. Since $L$ is an I-lattice there exists a $\mu \varepsilon I R(\sigma, L)$ st $\pi \leq \mu(L)$. Projecting upward $\hat{\mu} \varepsilon \operatorname{IR}(\sigma$, $W(\sigma, L))$ and $\hat{\pi} \leq \hat{\mu}(W(\sigma, L))$. Since $\mu \varepsilon S(\hat{\mu}), S(\hat{\pi}) \neq \emptyset$. Therefore $W(\sigma, L)$ is lindelof and thus so is $\tau \mathbf{W}(\sigma, \mathbf{L})$.

Conversely if $\tau \mathrm{W}(\sigma, \mathbf{L})$ is lindelof then so is $W(\sigma, L)$. Let $\pi \varepsilon \Pi(\sigma, L)$, then projecting upwards $\hat{\pi} \varepsilon \Pi(\sigma, W(\sigma, L))$ and $\hat{\pi}(W(\sigma, L))=\pi(L)$ LEL.Since $W(\sigma, L)$ is lindelof $S(\hat{\pi}) \neq \emptyset$ and there exists a $\mu \varepsilon S(\hat{\pi})$ st $\mu \varepsilon \operatorname{IR}(\sigma, \mathbf{L})$ and if $\hat{\pi}(\mathrm{W}(\sigma, \mathrm{L}))=\pi(\mathrm{L})=1 \mathrm{~L} \varepsilon \mathbf{L}$ then $\mu \varepsilon \mathrm{W}(\sigma, \mathrm{L})$ and $\mu(\mathrm{L})=1$. Thus $\pi \leq \mu(\mathbf{L})$ and $\mathbf{L}$ is an I-lattice.

THEOREM 4.2. Let $\mathbf{L}$ be an I-lattice, and also a delta lattice then $\operatorname{I}\left(\sigma^{*}, \mathbf{L}\right)=\operatorname{IR}(\sigma, \mathbf{L})$ implies $\mathbf{L}$ is complemented.

PROOF. Assume that $\mathbf{L}$ is not complemented then for some $L \varepsilon L L^{\prime} \notin \mathbf{L}$.Conside $F=\{\tilde{L}\} \tilde{L} \varepsilon \mathbf{L}$. $\left.\tilde{L} \supseteq L^{\prime}\right\}$, then $F$ has the finite intersection property and associated with $F$ is a filter $\pi \varepsilon \Pi(L)$. In addition, since $L$ is delta , then $\pi \varepsilon \Pi(\sigma, L)$ and $L^{\prime}$ is not cg (otherwise $L^{\prime}$ would belong to $L$, which would contradict the hypothesis). Since $L$ is an I-lattice there exists $\mu \varepsilon I R(\sigma, L)$ st $\pi \leq \mu(L)$ and since $I(\sigma *$, $\mathbf{L})=\operatorname{IR}(\sigma, \mathbf{L})$ then $\mu \varepsilon \operatorname{IR}\left(\sigma, \mathbf{L}^{\prime}\right)$ and $\mu\left(\mathbf{L}^{\prime}\right)=1$. But since $\mu \varepsilon \operatorname{IR}(\sigma, \mathbf{L}), \mu$ is associated with an $\mathbf{L}$. ultrafilter and thus $\mu(L)=1$. Thus $L$ is complemented.

We finally prove our last theorem in this section involving semi-separation, I-lattices and $\mu^{*}, \tilde{\mu}$.

THEOREM 4.3. Let $L_{1}, L_{2}$ be lattices of subsets of $X$ st $L_{2} \supseteq L_{1}, L_{1}$ a delta I-lattice, and for every $\mu \varepsilon I R\left(\sigma, L_{1}\right) \mu *\left(L_{2}\right)=\tilde{\mu}\left(L_{2}\right) L_{2} \varepsilon L_{2}$, then $L_{1}$ semi-separates $L_{2}$.

PROOF. Suppose $L_{1}$ did not semi-separate $L_{2}$ then there exists $L_{1} \varepsilon L_{1}, L_{2} \varepsilon L_{2}$ st $L_{1} \cap L_{2}=\emptyset$. but there does not exist a $L_{1} \sim \varepsilon L_{1}$ st $\tilde{L}_{1} \supseteq L_{2}$ and $L_{1} \cap \tilde{L}_{1}=\varnothing$.Look at $H=\left\{\tilde{L}_{1} \mid \tilde{L}_{1} \varepsilon L_{1}, \quad \tilde{L}_{1} L_{2}\right\}$ then $H$ has the finite intersection property and is a filter base and so can be extended to a filter.Since $L_{1}$ is delta, there exists $\pi \varepsilon \Pi(\sigma, L)$ associated with $H$.In addition since $L_{1}$ is an I-lattice there exists a $\mu \varepsilon \operatorname{IR}\left(\sigma, L_{1}\right)$ st $\pi \leq \mu$ on $L_{1}$.

Now look at $\mu *\left(\mathrm{~L}_{2}\right)=\tilde{\mu}\left(\mathrm{L}_{2}\right) . \tilde{\mu}\left(\mathrm{L}_{2}\right)=1$ since $\mu\left(\tilde{\mathrm{L}}_{1}\right)=1$ all $\tilde{\mathrm{L}}_{1} \varepsilon \mathrm{L}_{1}$ st $\tilde{\mathrm{L}}_{1} \supseteq \mathrm{L}_{2}$, thus $\mu *\left(\mathrm{~L}_{2}\right)=1$.In addition $\mu\left(L_{1}\right)=\mu *\left(L_{1}\right)=1$ since $L_{1}$ has non-empty intersection with $H, \mu$ is associated with an $L$ ultrafilter and the outer measure $\mu *=\mu$ restricted to $A\left(L_{1}\right)$. Thus $1=\mu *\left(L_{2}\right) \leq \mu *\left(L_{1}{ }^{\prime}\right)=\mu\left(L_{1}{ }^{\prime}\right)=0$, a contradiction. Therefore $\mathbf{L}_{1}$ semi-separates $\mathbf{L}_{2}$.

ACKNOWLEDGEMENT. I wish to thank the work and efforts of the referee's in reading this paper and greatly enhancing its presentation.

\section{REFERENCES}

1. A.D. ALEXSANDROV(ALEKSANDROV), Additive set functions in abstract spaces, (chapter 1), Mat. Sb. 8 (1940), 307-348.MR-315.

2. J. CAMACHO, JR., Extensions of lattice regular measures with applications, Jour. Indian Math. Soc., 54 (1989), 233-244.

3. G.M. EID, On normal lattices and Wallman spaces, Internat. J. Math. and Math. Sci, 13 No.1 (1990), 31-38.

4. Z.FROLIK, Prime filters with the cip, Comm. Math. Univ. Carolinae, Vol. 13 (1972), 553-575.

5. P. GRASSI, On subspaces of replete and measure replete spaces, Canad. Math. Bull. 27 (1) , (1984), 58-64.

6. C.C. HUERTA, Measure requirements on distributive lattices for Boolean algebras and topological applications, Proc. Amer. Math. Soc. 106 (12), 1989, 307-308.

7. M.SZETO, Measure repleteness and mapping preservations, Jour. Indian Math. Soc 43 (1979), 3552. 


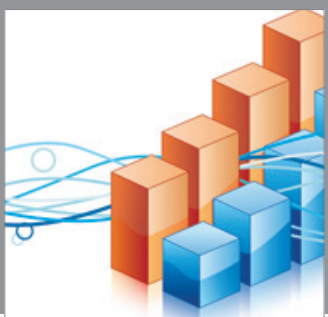

Advances in

Operations Research

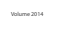

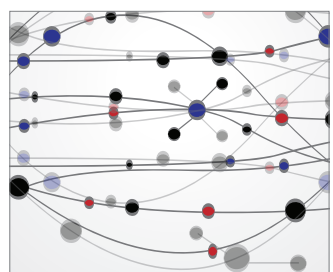

\section{The Scientific} World Journal
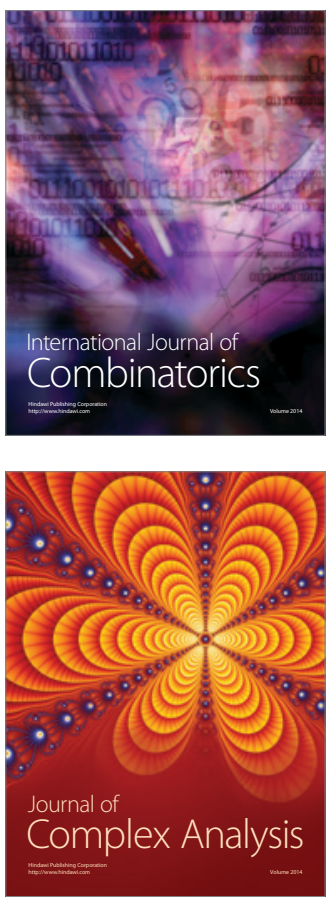

International Journal of

Mathematics and

Mathematical

Sciences
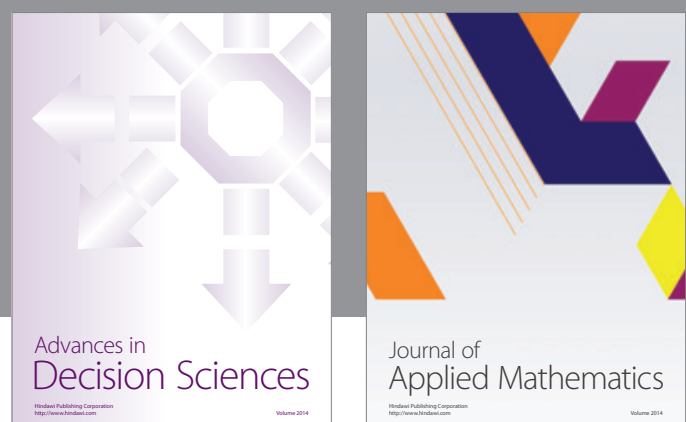

Journal of

Applied Mathematics
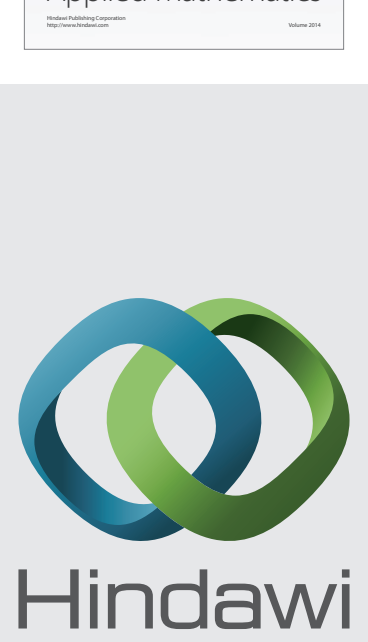

Submit your manuscripts at http://www.hindawi.com
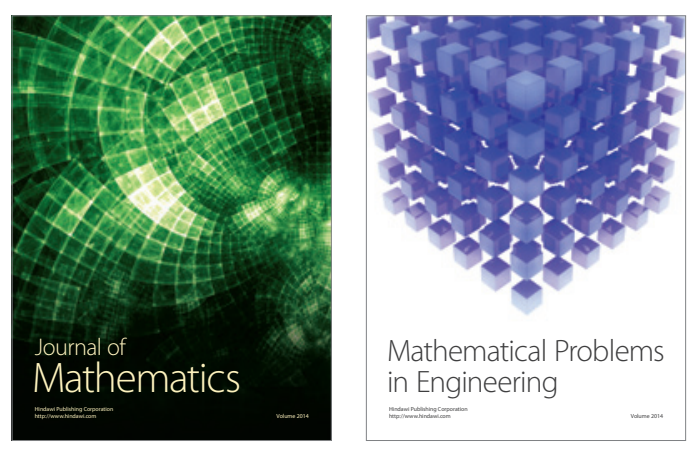

Mathematical Problems in Engineering
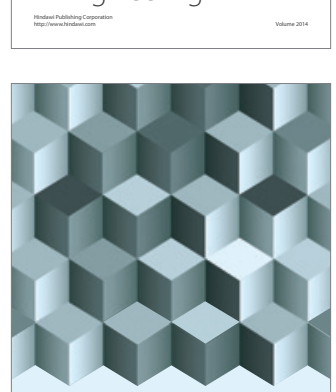

Journal of

Function Spaces
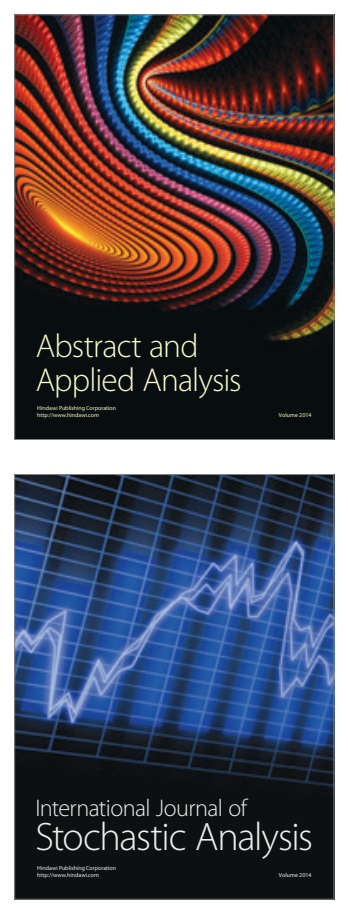

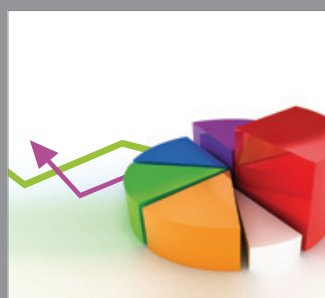

ournal of

Probability and Statistics

Promensencen
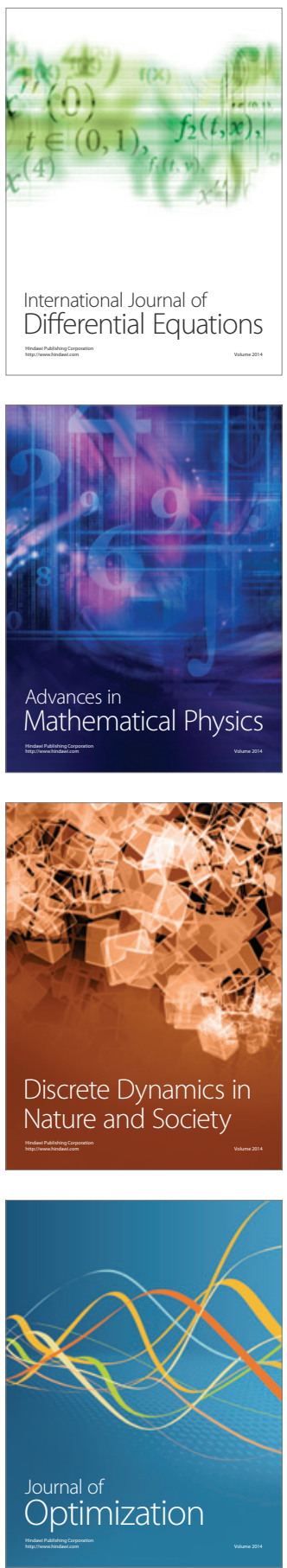